\title{
Tenofovir Is Superior to Entecavir on Tertiary Prevention for BCLC Stage 0/A Hepatocellular Carcinoma after Curative Resection
}

\author{
Ming-Chao Tsai ${ }^{a, b}$ Chih-Chi Wang ${ }^{c}$ Wei-Chen Lee ${ }^{d}$ Chih-Che Lin $^{b}$ \\ Kuo-Chin Chang ${ }^{\mathrm{a}}$ Chien-Hung Chen ${ }^{\mathrm{a}}$ Chao-Hung Hung ${ }^{\mathrm{a}}$ Ming-Tsung Lin ${ }^{\mathrm{a}}$ \\ Chang-Chun Hsiao ${ }^{e}$ Chao-Long Chen ${ }^{b}$ Rong-Nan Chien ${ }^{f}$ Tsung-Hui Hu ${ }^{a}$ \\ ${ }^{a}$ Division of Hepato-Gastroenterology, Department of Internal Medicine, Kaohsiung Chang Gung Memorial \\ Hospital and Chang Gung University College of Medicine, Kaohsiung, Taiwan; ${ }^{b}$ Graduate Institute of Clinical \\ Medical Sciences, College of Medicine, Chang Gung University, Taoyuan, Taiwan; 'Liver Transplantation Center \\ and Department of Surgery, Kaohsiung Chang Gung Memorial Hospital and Chang Gung University College of \\ Medicine, Kaohsiung, Taiwan; 'Division of Liver and Transplantation Surgery, Chang Gung Memorial Hospital and \\ Chang Gung University College of Medicine, Taoyuan, Taiwan; 'Division of Pulmonary and Critical Care Medicine, \\ Graduate Institute of Clinical Medical Sciences, College of Medicine, Kaohsiung Chang Gung Memorial Hospital, \\ Chang Gung University, Kaohsiung, Taiwan; 'Division of Hepato-Gastroenterology, Department of Internal \\ Medicine, Linko Chang Gung Memorial Hospital and Chang Gung University College of Medicine, Taoyuan, Taiwan
}

\section{Keywords}

Entecavir $\cdot$ Tenofovir disoproxil fumarate $\cdot$ Chronic hepatitis B $\cdot$ Hepatocellular carcinoma $\cdot$ Recurrence

\footnotetext{
Abstract

Background: It is unclear whether entecavir (ETV) and tenofovir disoproxil fumarate (TDF) have different effects on hepatocellular carcinoma $(\mathrm{HCC})$ recurrence and death in patients receiving curative hepatectomy for hepatitis $B$ virus (HBV)-related HCC. Aims: The aim of this study was to compare the long-term efficacy of ETV and TDF in HCC recurrence and overall survival (OS) of patients after curative hepatectomy. Methods: From January 2010 to December 2019, 20,572 patients with HCC who received hepatectomy were screened for study eligibility. Finally, a total of 219 consecutive patients treated with ETV $(n=146)$ or TDF $(n=73)$ after curative hepatectomy for HBV-related HCC of Barcelona Clinic Liver Cancer stage 0 or A were analyzed by propensity
}

score matching (PSM) (2:1) analysis and competing risk analysis. HCC recurrence and OS of patients were compared between ETV and TDF groups. Result: After a median follow-up of 52.2 months, 81 patients (37.0\%) had HCC recurrence, 33 (15.1\%) died, and 5 (2.3\%) received liver transplantation. TDF therapy was an independent protective factor for $\mathrm{HCC}$ recurrence compared with ETV therapy $(\mathrm{HR}, 1.687 ; 95 \% \mathrm{Cl}, 1.027-$ $2.770, p=0.039$ ); however, no difference in the risk of death or liver transplantation. Results were similar in competing risk analysis. We further found that TDF therapy was significantly associated with a lower risk of late recurrence (HR, $4.705 ; 95 \% \mathrm{Cl}, 1.763-12.558, p=0.002)$, but not in early recurrence. Conclusions: TDF therapy is associated with a significantly lower risk of HCC recurrence, especially of late recurrence, than ETV therapy among patients who undergo curative hepatectomy for HBV-related early-stage HCC.

(c) 2021 The Author(s).

Published by S. Karger AG, Basel
(C) 2021 The Author(s)

Published by S. Karger AG, Basel

This is an Open Access article licensed under the Creative Commons Attribution-NonCommercial-4.0 International License (CC BY-NC) (http://www.karger.com/Services/OpenAccessLicense), applicable to the online version of the article only. Usage and distribution for commercial purposes requires written permission. 


\section{Introduction}

Hepatocellular carcinoma (HCC) is the second most common cause of cancer-related deaths worldwide [1]. The high incidence of HCC in Asia compared to other regions of the world is related to the predominance of hepatitis B virus (HBV)-related HCC [2]. Although HCC surveillance of high-risk populations may improve early tumor diagnosis and treatment and reduce mortality rates, the clinical outcomes of patients receiving curative treatments still remain unsatisfactory due to the high rate of recurrence [3]. Around 70\% of patients with HCC who undergo potentially curative surgical resection develop recurrence within 5 years; tumor recurrence occurs most frequently within 2 years of resection [4]. Many factors are known to affect the risk of recurrence in HCC, including tumor size, alpha-fetoprotein (AFP), microvascular invasion, cirrhosis, and the viral replication status of HBV, hepatitis C virus, etc. [4-6]. Of these factors, hepatic viral load is the most clinically controllable. Among patients with HBV-related HCC, a higher HBV viral load has been reported to be a strong independent risk factor for recurrence after surgical resection $[7,8]$, and antiviral therapies may prevent recurrence and improve survival after resection $[9,10]$. However, antiviral therapies do not eliminate tumor recurrence, and approximately 40-60\% of patients with HCC treated with nucleos(t)ide analogs (NUCs) develop recurrence after resection $[4,10]$.

Entecavir (ETV) and tenofovir disoproxil fumarate (TDF) are currently recommended as first-line therapies for patients with chronic hepatitis $\mathrm{B}(\mathrm{CHB})$ due to their potent antiviral activity and high genetic barrier to resistance [11-13]. ETV and TDF suppress viral replication and have long-term efficacy for reducing the risk of HCC in patients with $\mathrm{CHB}[14,15]$; however, the relative risk reduction efficacy of the 2 drugs is currently under debate. A number of studies published in 2019 and 2020 indicated that TDF lowered the risk of HCC more effectively than ETV $[10,16,17]$. However, these findings were not supported by other reports $[17,18]$, possibly due to the fact that the studies assessed heterogeneous populations. In addition to the risk of developing HCC, it is not clear whether ETV and TDF have different effects on the risk of tumor recurrence in patients with HCC. To date, only one cohort study from Korea has suggested that TDF may be associated with a lower risk of HCC recurrence and a better overall survival (OS) than ETV [19]. However, due to the lack of head-to-head randomized controlled trials that directly compare the 2 drugs, additional studies are necessary to validate this result. Therefore, we aimed to compare ETV and TDF on the risk of HCC recurrence and mortality or liver transplantation in a propensity-matched, HBV-related HCC cohort that received curative hepatectomy.

\section{Materials and Methods}

\section{Patient Population}

We retrospectively reviewed the Chang Gung Research Database, which is derived from the largest private hospital system in Taiwan, from January 2010 to December 2019, and retrieved data of HCC patients $(n=20,572)$ (Fig. 1). A total of 5,896 patients with early-stage HCC (Barcelona Clinic Liver Cancer [BCLC] stage 0 or A) were studied. We excluded patients receiving nonsurgery $(n=$ $3,634)$, other modalities before resection $(n=1,081)$, receiving primary liver transplantation $(n=39)$, coinfection with hepatitis $C$ virus $(n=647),<3$ months of follow-up period after the surgery $(n=9)$, no NUC treatment after resection $(n=630)$, delayed $(\bigotimes 3$ months) NUC treatment after resection $(n=194)$, and previous treatment with NUCs other than ETV or TDF $(n=82)$. Finally, 431 consecutive patients who received curative hepatectomy for $\mathrm{HBV}$ related BCLC-0 or A HCC were enrolled in this study. Of them, 347 and 84 patients received ETV and TDF treatment, respectively. The selection of ETV or TDF treatment depended on the preference of the clinical physicians, and all cases received $0.5 \mathrm{mg}$ of ETV or $300 \mathrm{mg}$ of TDF once daily or vary according to the renal function. All patients continued their initial treatment regimen (i.e., ETV or TDF) during the study period, except patients who experienced adverse events or developed drug resistance. The patients who changed NUCs were censored at the time of the change.

This study complies with the standards of the Declaration of Helsinki and current ethical guidelines, and approval was obtained from the Ethics Committee of Chang Gung Memorial Hospital (approval number: 201900065B0; 201901103B0). Due to the retrospective design of the study, the written informed consent was waived according to the Institutional Review Board.

\section{Propensity Score Analysis}

Propensity score matching (PSM) was applied to reduce selection bias by equating the 2 groups. All possible clinicopathological covariates, including age, sex, body mass index (BMI), diabetes mellitus, smoking, liver cirrhosis, BCLC stage, tumor size, tumor number, histological grade, microvascular invasion, capsule invasion, and satellite nodule, which might have affected the outcomes, were included when conducting PSM. Using NCSS 10 Statistical Software (LLC, Kaysville, UT, USA), the greedy method was used for matching at a 2:1 ratio between the study groups with a caliper width 0.2 -fold the standard deviation of the propensity score between the study groups. The standardized mean difference was used to evaluate the covariate balance after PSM.

\section{Study Outcomes}

The primary outcome was recurrence-free survival (RFS), defined as the interval between the operation and the date of diagnosis of the first HCC recurrence. The secondary outcome was OS, defined as the interval between the date of surgery and death, liver transplantation, or date of last follow-up. The end date of the follow-up was on April 30, 2020. 
Fig. 1. Patient selection flow diagram (*PSM analysis was based on the following variables: age, sex, body mass index, diabetes mellitus, hypertension, alcohol drinking, smoking, liver cirrhosis, BCLC stage, tumor size, tumor number, histology grade, microvascular invasion, capsule invasion, and satellite nodule). PSM, propensity score matching; BCLC, Barcelona Clinic Liver Cancer; HCC, hepatocellular carcinoma; NUCs, nucleos(t)ide analogs; ETV, entecavir; TDF, tenofovir disoproxil fumarate.

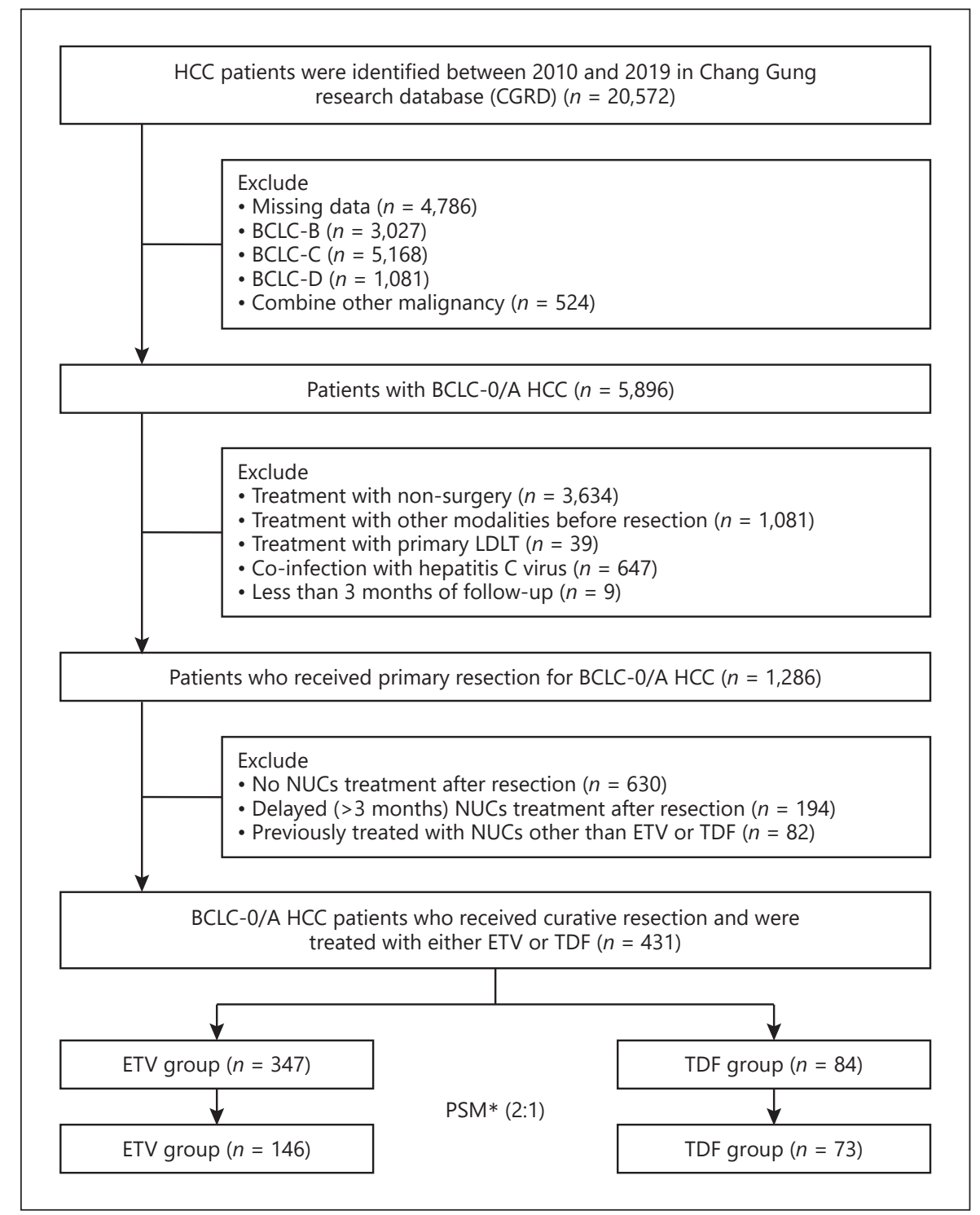

\section{Definition}

The diagnosis of HCC was assessed by contrast-enhanced multiphase computed tomography (CT) or magnetic resonance imaging (MRI), which fulfilled the criteria of the practice guidelines of the European Association for the Study of the Liver [20] or the American Association for the Study of Liver Disease [21], and was pathologically confirmed after the resection. The BCLC system is endorsed by the European Association for the Study of the Liver [22] and American Association for the Study of Liver Disease [23], and the revision of a single large $\mathrm{HCC}(>5 \mathrm{~cm})$ as BCLC stage B (instead of stage A) was widely adopted by Taiwan Liver Cancer Association (http://www.tlcaweb.org.tw/). Curative resection was defined as no viable tumor confirmed by contrast-enhanced CT or $\mathrm{MRI}$ within 3 months after resection. Intrahepatic $\mathrm{HCC}$ recurrence was defined using the same criteria as for the diagnosis of HCC with contrast-enhanced CT or MRI, showing typical features of HCC. Early recurrence was defined as recurrence within 2 years after $\mathrm{cu}$ - rative resection, while recurrence after 2 years of curative resection was defined as late recurrence [4]. We defined NUC treatmentnaïve as no NUC treatment 3 months before HCC diagnosis.

\section{Clinical Evaluations and Follow-Up}

The baseline demographics, serum biochemistry, and tumor characteristics were comprehensively recorded from the electronic medical records for analysis. HBV DNA (detection limit of 20 IU/mL, Roche COBAS TaqMan; Roche Molecular System, Branchburg, NJ, USA) was recorded at the time of within 1 month before the surgery. The histological features of the resected tumor, including satellite nodules, capsule invasion, microvascular invasion, and tumor differentiation, were recorded. Histological grade of tumor differentiation was scored using the modified nuclear grading scheme outlined by the Edmondson and Steiner, with tumor grade categorized as well, moderately, and poorly differentiated [24]. The grade for patients with multifocal disease is coded as the 
highest grade from any of the tumors. Degree of hepatic fibrosis in the nontumor part was scored according to the classification of Ishak et al. [25]. Liver cirrhosis was defined as Ishak et al. [25] fibrosis scores 5-6.

Patients were followed up at the 1st month after the surgery, followed by every 2-3 months by serum AFP levels, serum biochemistry, and abdominal ultrasound. Contrast-enhanced CT or MRI studies were identified if the tumor recurrence was suspected in the presence of elevation of serum AFP levels and ultrasonography detection of a new hepatic lesion. When HCC recurrence was confirmed, patients were hospitalized for multidisciplinary management, including liver resection, percutaneous ethanol injection, radiofrequency ablation, liver transplantation, transarterial embolization, chemotherapy, target therapy, immunotherapy, or supportive treatment.

Certain drugs, including aspirin and statin, which might affect the risk of HCC recurrence, were also analyzed. We collected the date of prescription, daily dose, and number of days supplied to defined daily dose (DDD) as recommended by the World Health Organization for measuring a prescribed amount of drugs [26]. Cumulative DDD (cDDD) was estimated as the sum of dispensed DDDs of any statins (namely Atorvastatin, Fluvastatin, Pitavastatin, and Rosuvastatin) and aspirin from 1 year before tumor resection to HCC recurrence. Patients taking >90 cDDD were enrolled in the use group.

\section{Statistical Analysis}

Continuous data are expressed as mean \pm standard deviation, and categorical data are expressed as number (percentage). Demographic data were compared between groups using the $\chi^{2}$ test or the Fisher's exact test, as appropriate. After creating the propensity score weight, the Kaplan-Meier method was used to generate cumulative incidence curves for HCC recurrence and death/liver transplantation. Analysis of prognostic factors for RFS and OS was performed using the Cox proportional hazards model. For multivariate analysis, based on the conservative thought, first, all potential risk factors ( $p<0.1$ in the univariate analysis) were required to be included in the multivariate analysis and demonstrated in model 1. Second, the multivariate analysis using the stepwise approach with $p<0.05$ as selection criterion was also revealed in model 2. A time-dependent, receiver-operating characteristic (ROC) curve analysis was used to assess the best cutoff point of BMI, AST, ALT, albumin, and AFP by Youden's index for predicting tumor recurrence and death/liver transplantation [27]. Since patients who died or underwent liver transplantation were no longer at risk for HCC recurrence, competing risk analyses were also conducted to evaluate the cumulative incidence of HCC recurrence, with death or liver transplantation considered a competing risk.

Statistical analyses were performed using SPSS 23.0 software (IBM, Chicago, IL, USA). All statistical tests were considered significant with 2 -sided and $p$ values $<0.05$.

\section{Results}

\section{Clinicopathological Characteristics of the Total Study Cohort}

Among a total of 431 patients included in this study, $347(80.5 \%)$ and $84(19.2 \%)$ patients received ETV and
TDF treatments, respectively. The mean age of the patients was 56.6 years, and the majority of patients were male $374(86.8 \%)$. The median size of HCC was $2.6 \mathrm{~cm}$ and all patients were in BCLC stage $0(n=119,27.6 \%)$ or A $(n=312,72.4 \%)$. Cirrhosis was histologically confirmed and was present in 252 (58.5\%) patients. The mean follow-up duration was $53.4 \pm 28.7$ months.

Before the PSM analysis, most characteristics were not significantly different between ETV and TDF groups except those patients in the ETV group were with a significantly higher proportion of NUC treatment before $\mathrm{HCC}$ resection ( $41.5 \%$ vs. $21.4 \%, p=0.001$ ), duration of NUC treatment before surgery $(27.4 \pm 16.8$ vs. $12.7 \pm 4.7$ months, $p<0.001)$, and a lower proportion of satellite nodule ( $2.6 \%$ vs. $10.7 \%, p=0.001)$ than TDF-treated patients (Table 1). After PSM, you can notice that most measured characteristics were balanced between ETV- and TDF-treated groups (standardarized mean differentce $<20 \%$ for selected variables), except for the proportion and duration of NUC treatment before surgery, which was due to the different approval dates of the 2 agents in Taiwan; ETV was first approved in August 2008 and TDF, in June 2011. Finally, we generated a 2:1 PS-matched analysis, including a total of 219 patients (146 patients on ETV and 73 patients on TDF), who had no significant differences in terms of all confounding factors for subsequent analysis, including the duration of follow-up period, which might be one of the most important variables for outcomes' comparison $(52.0 \pm 28.6$ vs. $52.5 \pm 24.0$ months, $p=0.890)$ (Table 1$)$.

\section{Risk Factors Associated with RFS}

A total of 81 (37.0\%) patients experienced HCC recurrence during the follow-up period, 59 (40.4\%) in the ETV group and $22(30.1 \%)$ in the TDF group. The 1-, 3-, and 5 -year RFS rates were $88.9 \%, 63.5 \%$, and $53.5 \%$, respectively, in the ETV group, while it was $86.1 \%, 75.8 \%$, and $66.1 \%$, respectively, in the TDF group ( $p=0.062$, Fig. $2 a$ ). According to the univariant Cox regression analysis, incidence of HCC recurrence was significantly higher in patients with DM $(p=0.045)$, current alcohol drinking ( $p=$ $0.022)$, current smoking ( $p=0.041)$, presence of liver cirrhosis $(p=0.007)$, BCLC A versus $0(p=0.017)$, larger tumor size $(p=0.006)$, presence of satellite nodule $(p=$ $0.019)$, and statin use ( $p=0.047)$ (Table 2).

In addition, based on the multivariable Cox regression analysis taking potential risk factors $(p<0.1$ on univariate analysis), presence of satellite nodule (hazard ratio [HR], 2.379; 95\% CI, 1.050-5.392, $p=0.038$ ), statin use (HR, 
Table 1. Patient characteristics before and after matching

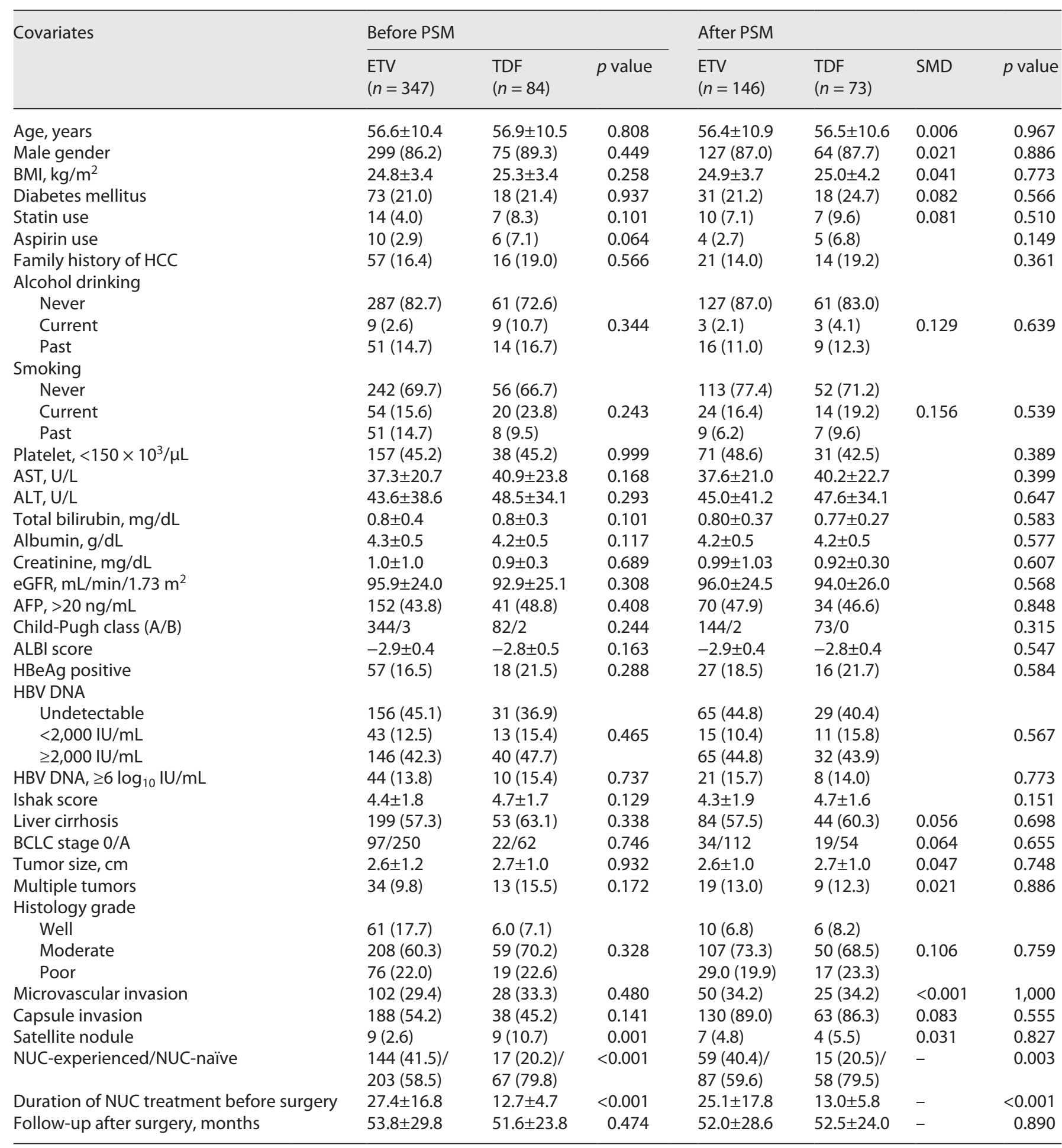

Data are expressed as mean \pm standard deviation or $n(\%)$. ETV, entecavir; TDF, tenofovir disoproxil fumarate; NUCs, nucleos(t)ide analogs; AST, aspartate aminotransferase; ALT, alanine aminotransferase; eGFR, estimated glomerular filtration rate; AFP, alpha-fetoprotein; $A L B I$, albumin-bilirubin; HBV, hepatitis B virus; HBeAg, hepatitis B e antigen; SMD, standardized mean difference. PSM, propensity score matching; BCLC, Barcelona Clinic Liver Cancer; HCC, hepatocellular carcinoma; NUCs, nucleos(t)ide analogs; BMI, body mass index. 


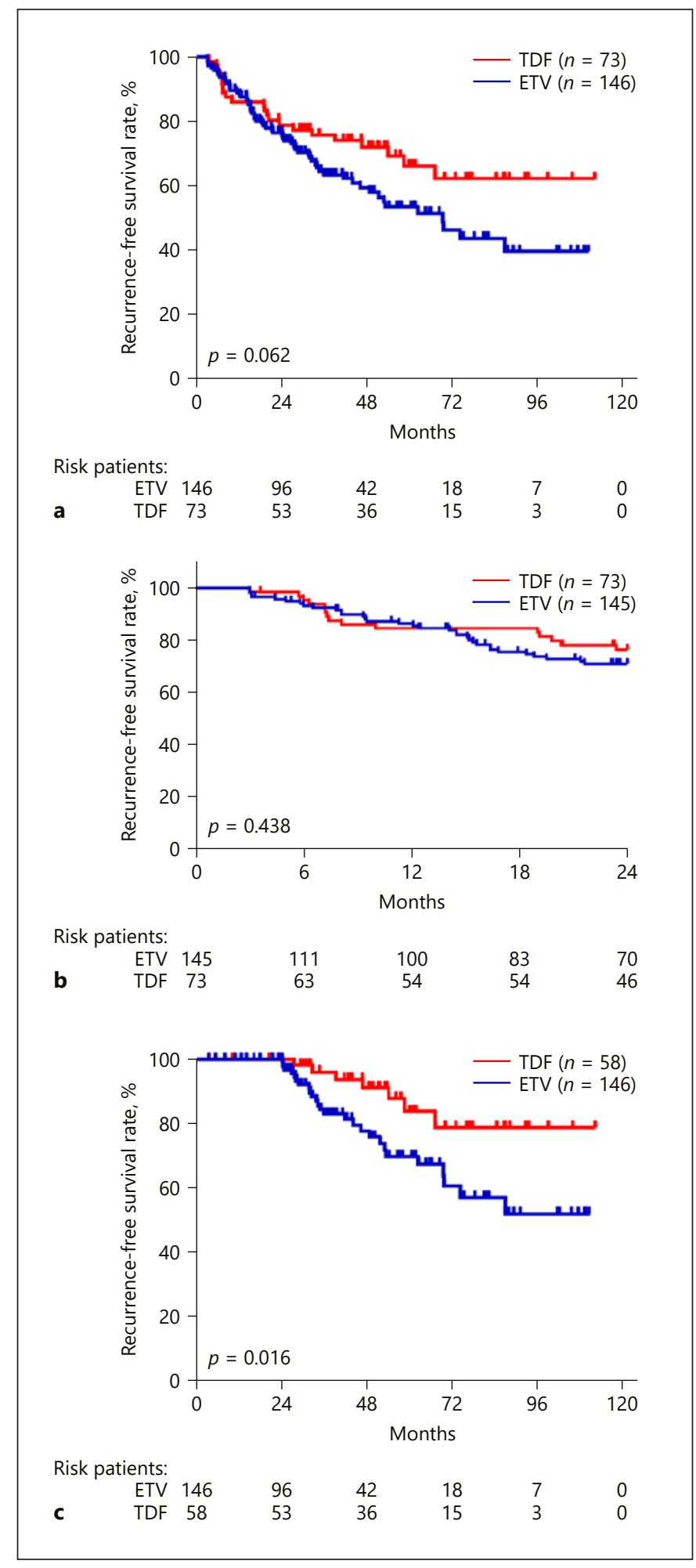

Fig. 2. RFS after curative resection in patients with HBV-related HCC under ETV or TDF treatment. The overall (a) for early RFS and (b) for late RFS (c). RFS, recurrence-free survival; HBV, hepatitis B virus; HCC, hepatocellular carcinoma; ETV, entecavir; TDF, tenofovir disoproxil fumarate.

TDF versus ETV on Tertiary HCC Prevention
0.118 ; $95 \% \mathrm{CI}, 0.016-0.879, p=0.037)$, and ETV treatment (HR, 1.805; 95\% CI, 1.074-3.033, $p=0.026$ ) were independent risk factors for RFS (Table 2 multivariable model 1). Using the stepwise selection approach, presence of cirrhosis (HR, 1.779; 95\% CI, 1.098-2.881, $p=$ $0.019)$, larger tumor size (HR, 1.247; 95\% CI, 1.027-1.515, $p=0.026)$, presence of satellite nodule (HR, 2.560; 95\% CI, 1.166-5.618, $p=0.019)$, and ETV versus TDF treatment (HR, 1.687; 95\% C, 1.027-2.770, $p=0.039$ ) were independent risk factors for RFS (Table 2 multivariable model 2).

During the observation, 11 patients died $(n=7)$ or received liver transplantation $(n=4)$ before the HCC recurrence. With the competing risk analysis, ETV treatment was again associated with a significantly higher risk of HCC recurrence than TDF treatment (HR, 1.702; 95\% CI, 1.037-2.793, $p=0.035$; online suppl. Table 1; see www. karger.com/doi/10.1159/000518940 for all online suppl. material; online suppl. Fig. 1).

\section{Risk Factors Associated with Early and Late HCC \\ Recurrence}

HCC recurrence is typically classified as early or late recurrence, which is generally defined using a cutoff of 2 years after surgery $[4,28]$. Among the total of 81 patients with HCC recurrence, 48 (59.3\%) and 33 (40.7\%) patients had early ( $<2$ years) and late ( $₫ 2$ years) HCC recurrences, respectively: in early recurrence, $33(22.6 \%)$ in the ETV group and 15 (20.5\%) in the TDF group ( $p=0.729)$; in late recurrence, $26(23.0 \%)$ in the ETV group and $7(12.2 \%)$ in the TDF group $(p=0.086)$. As shown in Figure $2 b$, the Kaplan-Meier method showed that there was no significant difference in early HCC recurrence between the ETV and TDF groups; however, ETV treatment was significantly associated with a higher risk of late recurrence than TDF therapy (Fig. 2c, $p=$ $0.016)$. Similarly, the multivariate analysis revealed that ETV treatment was an independent risk factor of late recurrence (HR, 2.792, $p=0.019$ in model 1 ; HR, 4.705; $p=0.002$ in model 2$)$ when compared with TDF treatment (Table 4).

Other factors that were significantly associated with a higher risk of early recurrence were presence of satellite nodule (HR, 3.072; 95\% CI, 1.212-7.784, $p=0.018$ ) and presence of liver cirrhosis (HR, 2.060; 95\% CI, 1.104$3.844, p=0.023$ ) (Table 3). For late recurrence, family history of HCC (HR, 2.412; 95\% CI, 1.050-5.542, $p=$ $0.037)$ and current smoking (HR, 2.720; 95\% CI, 1.188$6.224, p=0.018$ ) were significantly associated with late recurrence in the multivariate analysis (Table 4).

Liver Cancer 2022;11:22-37

DOI: $10.1159 / 000518940$ 
Table 2. Prognostic factors associated with HCC recurrence

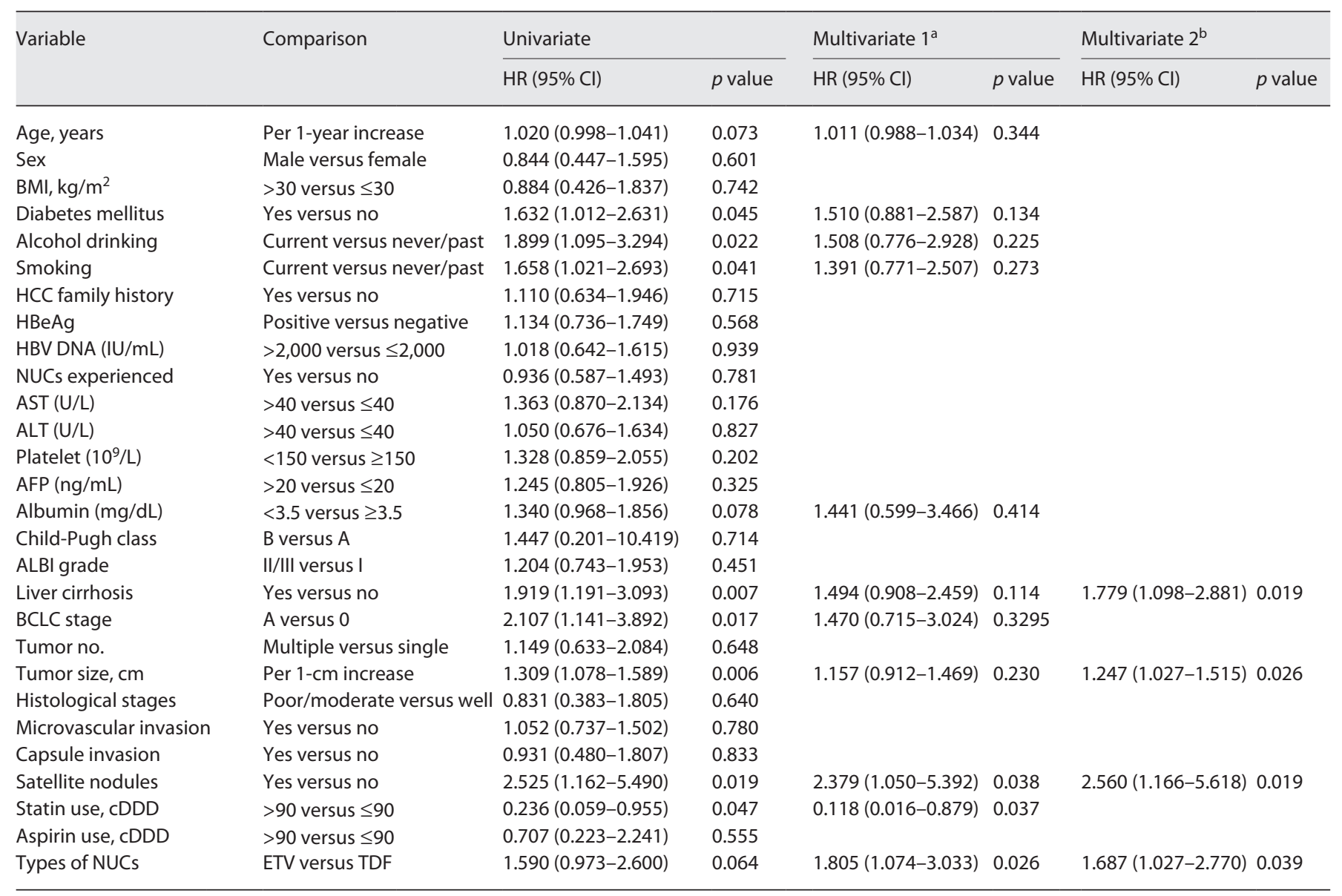

$\mathrm{HR}$, hazard ratio; $\mathrm{Cl}$, confidence interval; $\mathrm{BMI}$, body mass index; ETV, entecavir; TDF, tenofovir disoproxil fumarate; cDDD, cumulative defined daily doses; AST, aspartate aminotransferase; ALT, alanine aminotransferase; AFP, alpha-fetoprotein; ALBI, albumin-bilirubin; HCC, hepatocellular carcinoma; HBeAg, hepatitis B e antigen; NUCs, nucleos(t)ide analogs; HBV, hepatitis B virus; BCLC, Barcelona Clinic Liver Cancer. ${ }^{a}$ Multivariate 1: multivariable analysis based on potential risk factors ( $p<0.1$ in the univariate Cox model). ${ }^{b}$ Multivariate 2 : multivariable analysis with variables $(p<0.1$ in the univariate Cox model) adjustment based on the stepwise selection.

\section{Risk Factors Associated with OS}

A total of $33(15.0 \%)$ patients died and $5(2.3 \%)$ patients received liver transplantation during the follow-up period, 30 in the ETV group and 8 in the TDF group. Overall transplant-free survival rates at 1,3 , and 5 years were $98.6 \%, 89.6 \%$, and $75.3 \%$, respectively, in the ETV group, while it was $97.3 \%, 91.3 \%$, and $88.0 \%$, respectively, in the TDF group ( $p=0.104$, Fig. 3 ).

By the stepwise Cox proportional hazard model, presence of cirrhosis (HR, 4.275; 95\% CI, 1.659-11.014, $p=$ $0.003)$ and BCLC stage A versus 0 (HR, 3.431; 95\% CI, $1.053-11.180, p=0.041$ ) were independent risk factors for death or liver transplantation (Table 5). In contrast, there was no significant difference for death or liver transplantation between ETV and TDF treatment. When death was considered the endpoint and liver transplantation a censoring event, results were also similar (data not shown).

\section{Risk Factors Associated with RFS for ETV-or TDF \\ Treatment-Naïve Patients after Surgery}

In the subgroup analysis of 145 (66.2\%) NUC treatment-naïve patients after surgery, 87 of whom were ETVnaïve and 58 were TDF-naïve, respectively, and the TDF group had significantly better RFS than the ETV group $(p=0.003$, Fig. $4 a)$. There was no significant difference in early RFS between the ETV- and TDF-naïve groups (Fig. 4b), though the TDF-naïve group had better late RFS ( $p=0.008$, Fig. $4 c)$. In multivariable analysis, HCC recurrence was independently associated with ETV treatment
28

Liver Cancer 2022;11:22-37 DOI: $10.1159 / 000518940$
Tsai/Wang/Lee/Lin/Chang/Chen/Hung/ Lin/Hsiao/Chen/Chien/Hu 


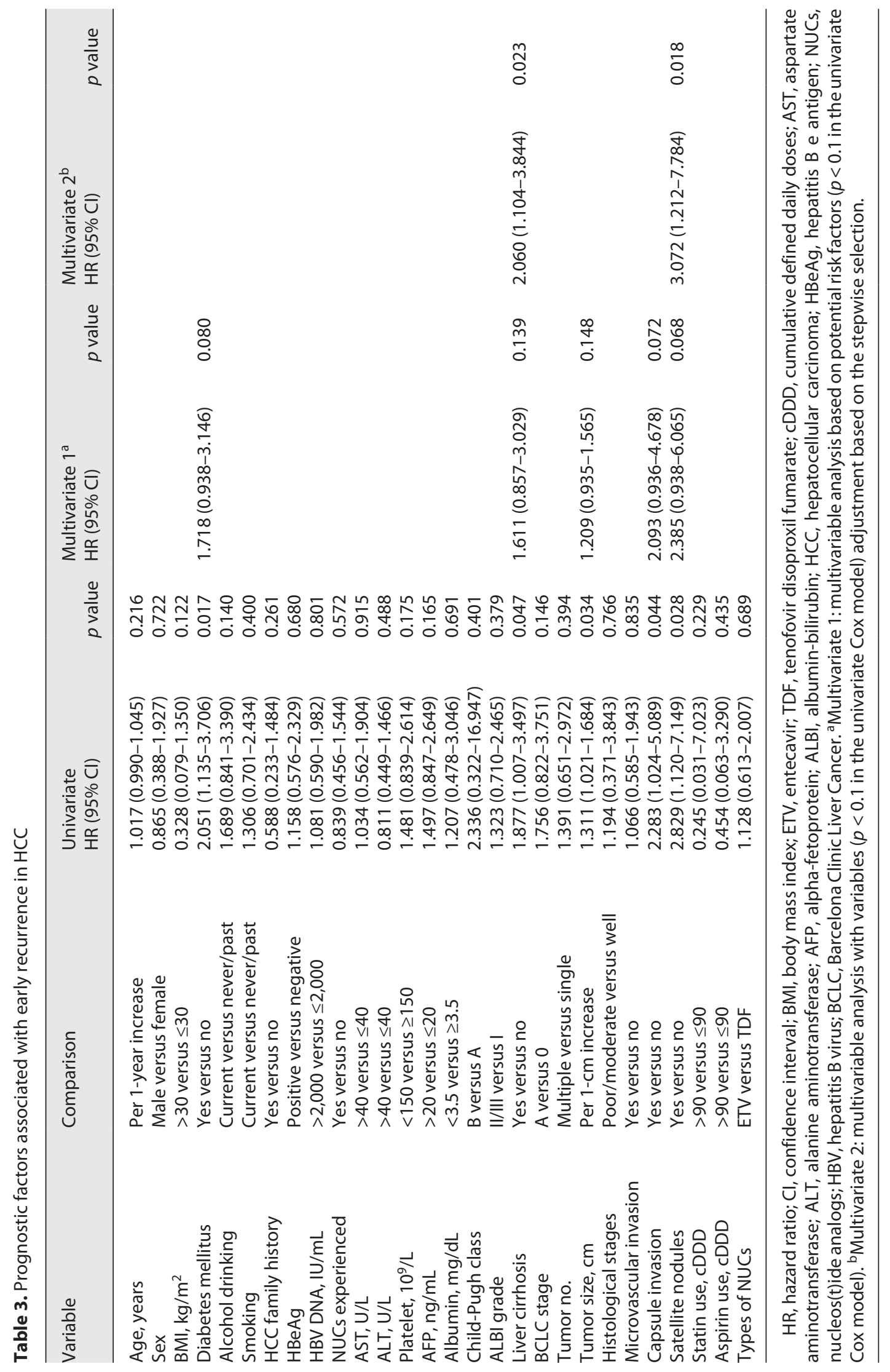


Table 4. Prognostic factors associated with late recurrence in HCC

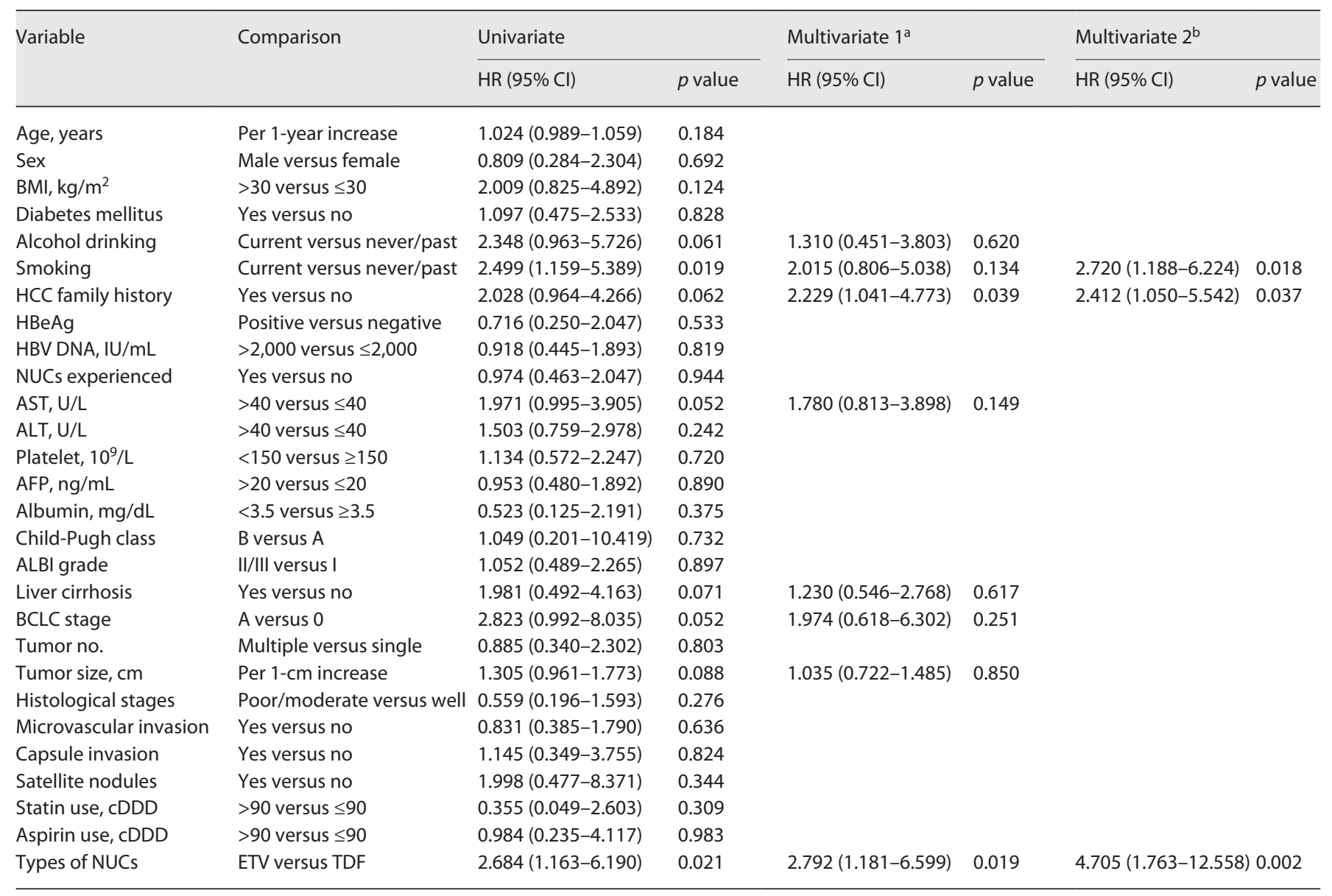

$\mathrm{HR}$, hazard ratio; $\mathrm{Cl}$, confidence interval; BMI, body mass index; ETV, entecavir; TDF, tenofovir disoproxil fumarate; cDDD, cumulative defined daily doses; AST, aspartate aminotransferase; ALT, alanine aminotransferase; AFP, alpha-fetoprotein; ALBI, albumin-bilirubin; HCC, hepatocellular carcinoma; HBeAg, hepatitis B e antigen; NUCs, nucleos(t)ide analogs; HBV, hepatitis B virus; BCLC, Barcelona Clinic Liver Cancer. ${ }^{a}$ Multivariate 1: multivariable analysis based on potential risk factors ( $p<0.1$ in the univariate Cox model). ${ }^{\mathrm{b}}$ Multivariate 2 : multivariable analysis with variables $(p<0.1$ in the univariate Cox model) adjustment based on the stepwise selection.

(HR, 2.548; 95\% CI, 1.382-4.697, $p=0.003$ ), presence of liver cirrhosis (HR, 1.956; 95\% CI, 1.079-3.546, $p=0.027$ ), and presence of satellite nodules (HR, 2.846; 95\% CI, $0.999-8.111, p=0.050)$ in the NUC-naïve cohort after surgery (online suppl. Table 2).

\section{On-Therapy Response after ETV or TDF Treatment}

Patients treated with ETV and patients treated with TDF had similar rates of on-therapy biochemical and virological responses at 1 or 2 years after resection (Table 6). The probability of reaching undetectable HBV DNA was not significantly different between the ETVand TDF-treated patients at 1 year $(81.4 \%$ vs. $85.0 \%, p=$ $0.553)$ or 2 years $(82.0 \%$ vs. $85.2 \%, p=0.615)$ after resection. We also evaluated the on-therapy factors associated with HCC recurrence. Both univariate and multivariate Cox models indicated that ALT normalization, albumin, and undetectable HBV DNA at 1 year or 2 years after surgery were not significant prognostic factors for HCC recurrence. Therefore, we conclude that issue on the onset of antivirals did not impact the recurrence of HCC after resection in this study.

\section{Discussion}

This multicenter, PS-matched cohort study explored the comparative risk reduction effects of ETV and TDF on HCC recurrence, mortality, and LT in HBV-related early-stage HCC after curative hepatectomy. TDF was as-
30

Liver Cancer 2022;11:22-37

DOI: $10.1159 / 000518940$
Tsai/Wang/Lee/Lin/Chang/Chen/Hung/ Lin/Hsiao/Chen/Chien/Hu 
Table 5. Prognostic factors associated with overall mortality/liver transplantation

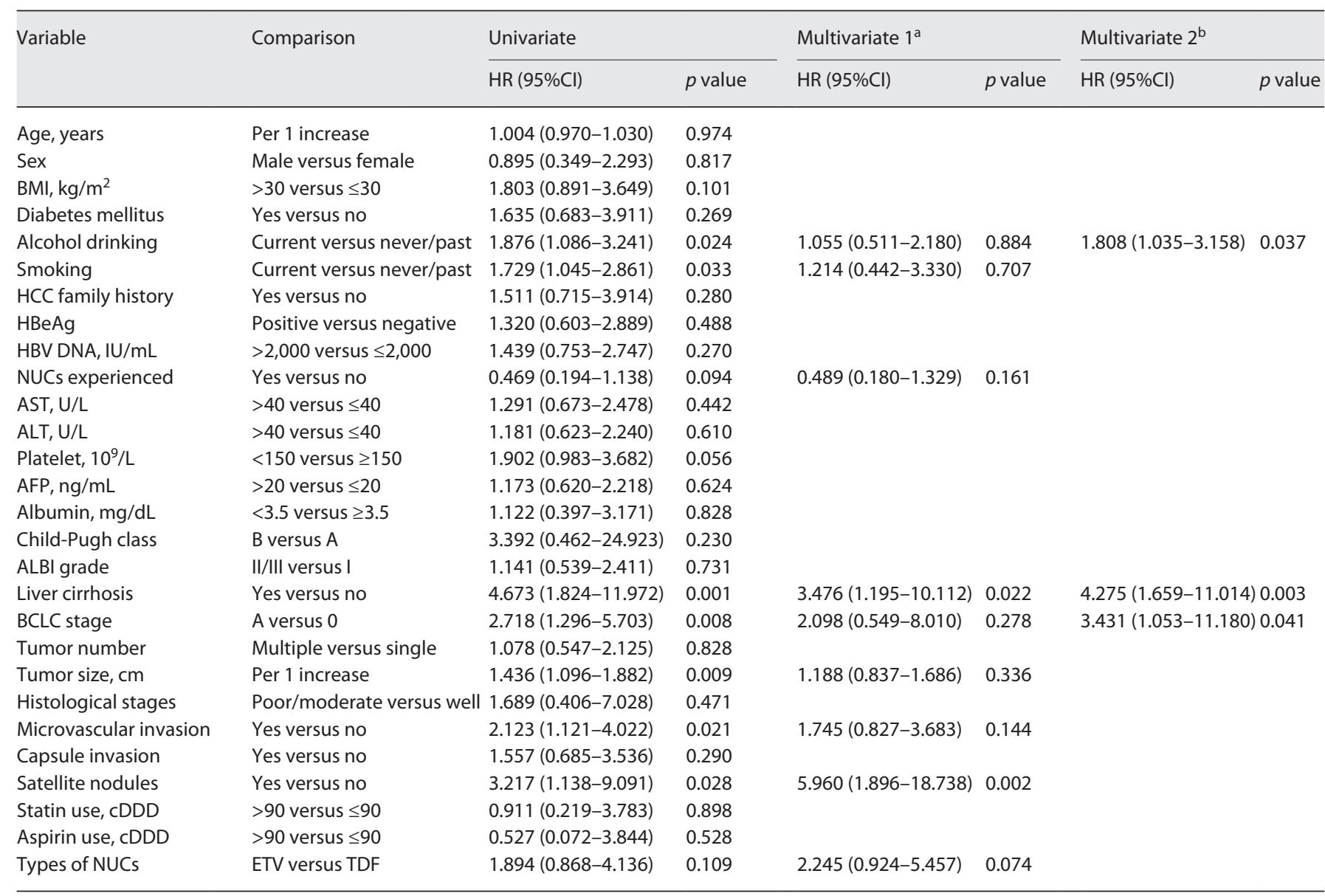

$\mathrm{HR}$, hazard ratio; $\mathrm{Cl}$, confidence interval; BMI, body mass index; ETV, entecavir; TDF, tenofovir disoproxil fumarate; CDDD, cumulative defined daily doses; AST, aspartate aminotransferase; ALT, alanine aminotransferase; AFP, alpha-fetoprotein; ALBI, albumin-bilirubin; HCC, hepatocellular carcinoma; HBeAg, hepatitis B e antigen; NUCs, nucleos(t)ide analogs; HBV, hepatitis B virus; BCLC, Barcelona Clinic Liver Cancer. ${ }^{a}$ Multivariate 1: multivariable analysis based on potential risk factors $\left(p<0.1\right.$ in the univariate Cox model). ${ }^{\mathrm{b}}$ Multivariate 2 : multivariable analysis with variables $(p<0.1$ in the univariate Cox model) adjustment based on the stepwise selection.

sociated with a significantly lower rate of HCC recurrence than ETV. Furthermore, we found that the difference between 2 agents was on the late recurrence, not on the early recurrence. To the best of our knowledge, this is the first study to indicate that TDF is specifically associated with late recurrence in patients with HCC after resection.

The results of this study were similar but not exactly the same with a recently published article from Korea [19]. Choi et al. [19] enrolled the largest cohort of patients with HBV-related BCLC stage 0 or A HCC receiving curative hepatectomy, which included 813 patients treated with ETV and 882 patients treated with TDF. Their study found that TDF was an independent protective factor for both early and late tumor recurrence in HCC. However, this result was unexpected, especially in the context of early recurrence. Early tumor recurrence (within 2 years after surgery) is generally accepted to be associated with primary tumor-related factors, including the presence of vascular or capsule invasion, satellite nodules, and poor histological grade. In contrast, late recurrence ( $>2$ years after surgery) is proposed to be the result of de novo recurrence, which has been associated with the remaining liver and closely linked to the "field effect," including viral loads, inflammatory activity, and the degree of fibrosis [4, 28]. Thus, the existing evidence supports the suggestion that antiviral therapies exert a tertiary preventative effect in HCC, especially for late tumor recurrence. In other words, antiviral therapies have a relatively low effect on 


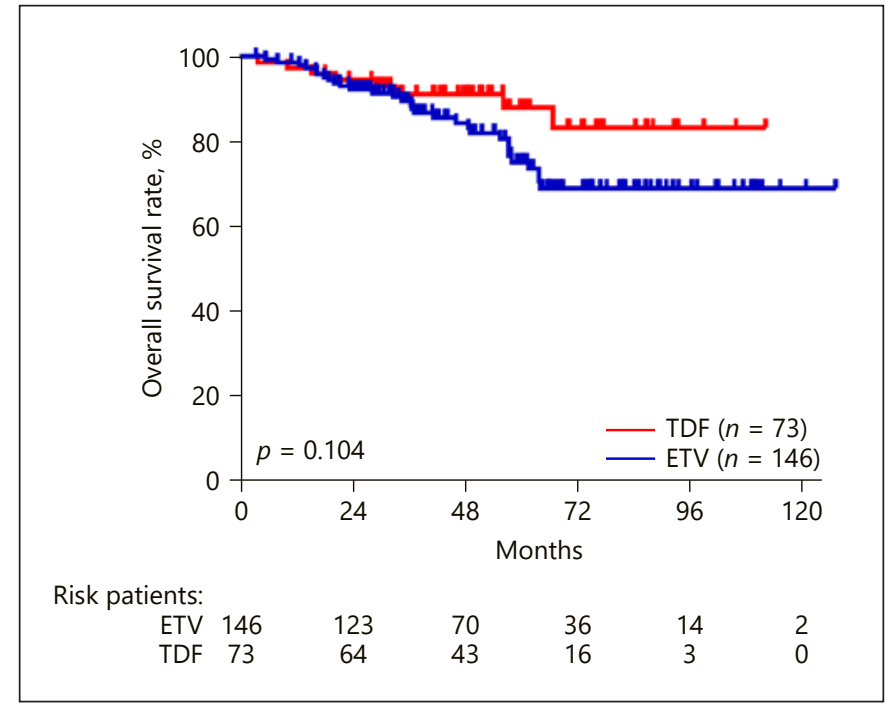

Fig. 3. OS after curative resection in patients with HBV-related HCC under ETV or TDF treatment. HBV, hepatitis B virus; HCC, hepatocellular carcinoma; ETV, entecavir; TDF, tenofovir disoproxil fumarate; OS, overall survival.

the prevention of early HCC recurrence. However, in the study by Choi et al., it is notable that the RFS rates of the TDF and ETV groups started to diverge at 1 year after surgery, and that means only 1 year of TDF treatment led to a $21 \%$ greater reduction $(\mathrm{HR}=0.79)$ in early HCC recurrence (i.e., in the second year) than ETV. Although the rapid decrease on recurrence resulting in a significant lower early recurrence in the TDF treatment than ETV treatment was first observed in that study, some potential factors, such as chemopreventive agents (statin, aspirin, etc.), and viral mutations, which have been reported to associate with HCC recurrence, were not analyzed. To overcome this discrepancy, prospective randomized clinical trials are needed to directly compare the effect of ETV and TDF on tumor recurrence in HCC. However, such studies are unlikely to be conducted in the near future. Therefore, our observational study has a considerable scientific value.

Another study recently published from Taiwan showed no significant differences in early and late tumor recurrence and OS between patients with HCC treated with ETV and TDF [29]. However, that study enrolled a relatively small number of patients treated with TDF $(n=42)$, and only 3 quarters of the patients had BCLC stage 0 or A (256/339). Notably, TDF was associated with a lower rate of late recurrence than ETV, although the difference was not significant $(\mathrm{HR}=0.324,95 \% \mathrm{CI}, 0.079-1.321, p=$

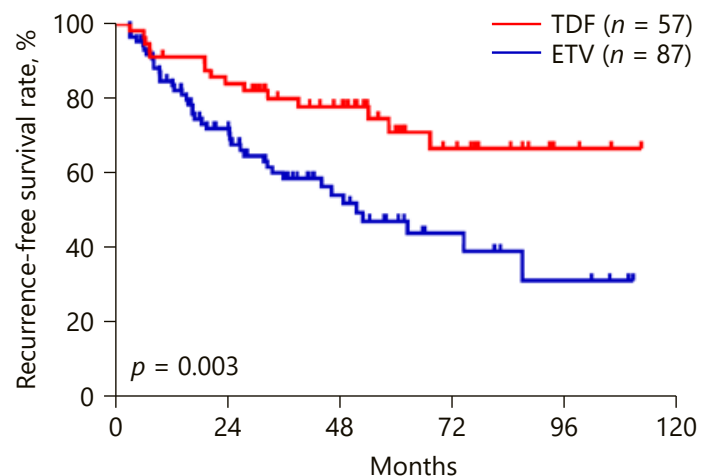

Risk patients:

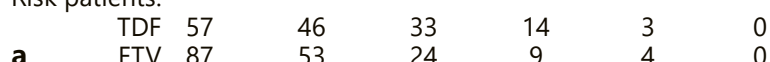

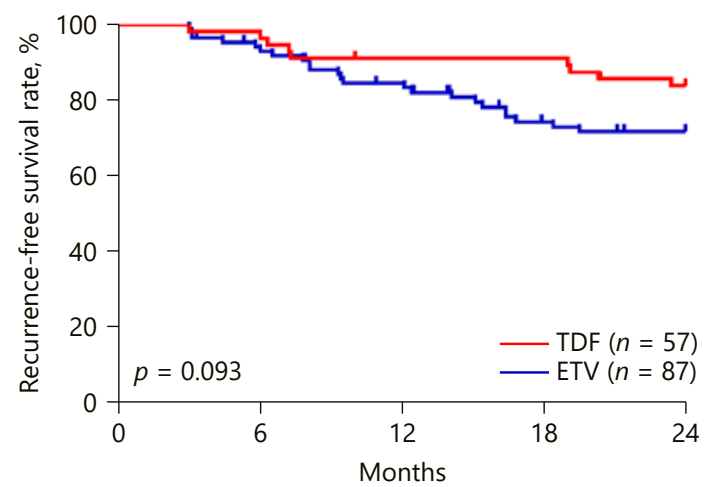

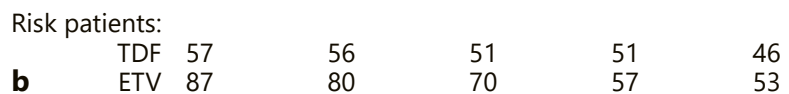

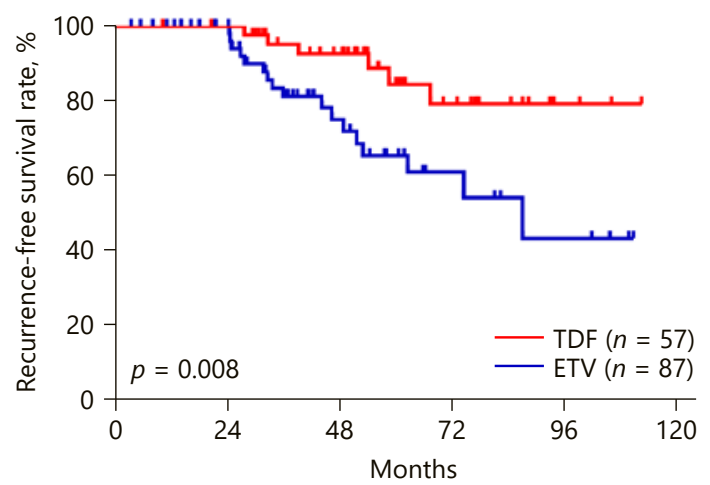

Risk patients:

$\begin{array}{cccccccc} & \text { TDF } & 57 & 46 & 33 & 114 & 3 & 0 \\ \text { C } & \text { ETV } & 87 & 53 & 24 & 9 & 4 & 0\end{array}$

Fig. 4. Recurrence-free survival of the propensity score-matched cohort of patients who were naive treated with either ETV or TDF after curative resection for HBV-related HCC. The overall (a) for early RFS (b) for late RFS (c). ETV, entecavir; TDF, tenofovir disoproxil fumarate; HBV, hepatitis B virus; HCC, hepatocellular carcinoma; RFS, recurrence-free survival.
Tsai/Wang/Lee/Lin/Chang/Chen/Hung/ Lin/Hsiao/Chen/Chien/Hu 
Table 6. Comparison of on-therapy response among patients with CHB-related early-stage HCC who were treated with either ETV or TDF after curative resection

\begin{tabular}{llll}
\hline & ETV $(n=146)$ & TDF $(n=73)$ & $p$ value \\
\hline At year 1 & & & 0.416 \\
AST $<40 \mathrm{U} / \mathrm{L}$ & $111 / 136(81.6)$ & $53 / 69(76.8)$ & 0.316 \\
ALT $<40 \mathrm{U} / \mathrm{L}$ & $107 / 137(78.1)$ & $51 / 71(71.8)$ & 0.480 \\
Total bilirubin, $\mathrm{mg} / \mathrm{dL}$ & $0.9 \pm 0.5$ & $0.9 \pm 0.3$ & 0.370 \\
Platelet, $<150 \times 10^{3} / \mu \mathrm{L}$ & $56 / 105(53.5)$ & $18 / 40(45.0)$ & 0.480 \\
Albumin, $\mathrm{g} / \mathrm{dL}$ & $4.4 \pm 0.4$ & $4.4 \pm 0.5$ & 0.191 \\
eGFR, $\mathrm{mL} / \mathrm{min} / 1.73 \mathrm{~m}^{2}$ & $87.9 \pm 25.8$ & $83.1 \pm 22.8$ & 0.553 \\
HBV DNA undetectable & $92 / 113(81.4)$ & $51 / 60(85.0)$ & 0.670 \\
At year 2 & & & 0.987 \\
AST $<40 \mathrm{U} / \mathrm{L}$ & $99 / 119(83.2)$ & $50 / 62(80.6)$ & 0.799 \\
ALT $<40 \mathrm{U} / \mathrm{L}$ & $92 / 120(76.7)$ & $49 / 64(76.6)$ & 0.202 \\
Total bilirubin, $\mathrm{mg} / \mathrm{dL}$ & $0.9 \pm 0.4$ & $0.9 \pm 0.3$ & 0.490 \\
Platelet, $<150 \times 10^{3} / \mu \mathrm{L}$ & $50 / 90(55.6)$ & $15 / 35(42.9)$ & 0.409 \\
Albumin, g/dL & $4.4 \pm 0.3$ & $4.3 \pm 0.5$ & 0.553 \\
eGFR, $\mathrm{mL} / \mathrm{min} / 1.73 \mathrm{~m}{ }^{2}$ & $83.3 \pm 22.7$ & $80.2 \pm 25.7$ & $46 / 54(85.2)$ \\
HBV DNA undetectable & $82 / 100(82.0)$ & & \\
\hline
\end{tabular}

Data are expressed as mean \pm standard deviation or $n / N(\%)$. ETV, entecavir; TDF, tenofovir disoproxil fumarate; AST, aspartate aminotransferase; ALT, alanine aminotransferase; eGFR, estimated glomerular filtration rate; HBV, hepatitis B virus.

0.116). This may be due to the small number of patients, and we propose that the difference may have been significant if more patients had been included in the TDF group.

In previous comparisons of ETV and TDF in treatment-naïve patients with $\mathrm{CHB}$ or patients with HBV-related HCC, the patients treated with ETV tended to be older and have more advanced diseases, a larger burden of comorbidities, and a longer follow-up duration than the TDF-treated patients, probably reflecting "patient warehousing." Severely ill patients were likely to have received ETV when it was first approved, whereas healthier patients were eligible for TDF treatment when it subsequently became available [30]. In addition, TDF may have been avoided in elderly patients due to concerns about renal dysfunction and decreased bone density. These biases are difficult to overcome, especially in real-world clinical practice. Therefore, most studies observed a higher incidence of HCC development in the ETV-treated cohorts before adjusted analysis, whereas no significant difference was observed after PSM. However, in the present study, most baseline characteristics were similar between the ETV and TDF groups, except for NUC treatment before HCC development and the presence of satellite nodules. This might be explained by the fact that patients with BCLC stage 0 or A HCC who are eligible to receive cura-

TDF versus ETV on Tertiary HCC Prevention tive resection have a better renal function than those without HCC and eligible for NUC treatment, 95.4 \pm 25.0 $\mathrm{mL} / \mathrm{min} / 1.73 \mathrm{~m}^{2}$ in the present study versus $87.7 \pm 27.0$ $\mathrm{mL} / \mathrm{min} / 1.73 \mathrm{~m}^{2}$ in our recent published study [18]; thus, physicians may not have a strong preference for either ETV or TDF treatment in these patients. Even so, we still employed PSM to account for the nonrandom assignment of the patients. Thus, our analysis in the context of low selection bias for ETV and TDF and adjustment for well-known risk factors for HCC recurrence, especially the duration of follow-up time between ETV and TDF, which might be the greatest impact on the difference of HCC occurrence in many meta-analyses [31], suggests that TDF leads to a lower rate of late tumor recurrence in HCC than ETV.

Another issue is whether NUC experience (treated with NUCs before HCC diagnosis) and NUC naïve (no treatment before HCC) affect recurrence in HCC. In the present study, $74(33.8 \%)$ and $145(66.2 \%)$ patients were NUC-experienced and -naïve, respectively. We found no significant difference in the incidence of $\mathrm{HCC}$ recurrence between NUC-experienced and NUC-naïve patients (online suppl. Fig. 2). We further excluded the NUC-experienced patients to only compare the ETV- and TDF-naïve treatment groups after HCC curative resection, to more realistically assess the effects of ETV and TDF on HCC 
recurrence. Similar to the entire cohort analysis, TDF treatment was associated with better RFS than ETV treatment (HR, 2.548; 95\% CI: 1.382-4.697, $p=0.003$; online suppl. Table 2). Furthermore, in the subgroup of NUCexperienced patients $(n=74)$, there was no significant difference in RFS between the ETV- and TDF-experienced groups (online suppl. Fig. 3), which may be due to the low numbers of patients in the TDF group $(n=15)$. It is worth noting that the duration of NUC treatment before HCC diagnosis was not associated with HCC recurrence, which implies that a longer duration of treatment before HCC diagnosis does not decrease the risk of HCC recurrence.

Although previous studies demonstrated that ETV and TDF are similarly effective in terms of HBV DNA suppression [32], other studies found that TDF was superior to ETV in patients with $\mathrm{CHB}$ with a high viral load [33]. This raises the question of whether the significant difference in HCC recurrence between ETV and TDF treatment was due to varied HBV DNA suppression. In the present study, there were no significant differences in the virological response rates between the ETV and TDF groups at 1 and 2 years after surgery, which indicates that the differences in HCC recurrence between patients treated with ETV and TDF are not due to altered HBV DNA suppression. Although the TDF group had a higher HBV DNA undetectable rate than the ETV group in the high viral load subgroup (HBV DNA $\geq 6 \log _{10}$ IU/ $\mathrm{mL}$ ), inadequate numbers of patients were followed up (only 21 and 8 patients with high HBV DNA in the ETV and TDF groups, respectively) to allow meaningful interpretations.

Debate still remains on whether ETV or TDF is more effective to reduce the risk of HCC among patients with $\mathrm{CHB}$. In terms of secondary prevention, it is well recognized that HCC has a low rate of occurrence rate under first-line NUCs (ETV or TDF); thus, assessment of a large cohort with an adequate and similar follow-up periods is necessary to elucidate the difference of effect of both NUCs. Actually, there is a lack of study to fully fit the criteria. There were always retrospective studies with noncirrhotic patients (low incidence rate of HCC), less TDF patients, and shorter follow-up periods of TDF in those studies. In contrast to secondary prevention, tertiary prevention is mainly administered to patients who have a high relative risk for HCC recurrence after surgical resection. Hence, we believe that it is easier to compare the difference of the effects from ETV and TDF under a higher incidence rate of events in this tertiary prevention study. In addition, the equivalent follow-up periods between
TDF and ETV groups would further minimize the bias, which existed in most of the secondary studies.

However, we cannot fully explain the mechanisms that underlie the potentially differential effects of ETV and TDF on HCC recurrence observed in the current study. Recent studies found some factors that may possibly explain these differences, including the anticancer effects of IFN- $\lambda 3$ induced by TDF [34], the association of ETV with carcinogenic chromosomal aberrations [35], and the higher rate of regression of cirrhosis after long-term TDF treatment than ETV treatment (74\% vs. 62\%) [15]. Recently, quantification of HBsAg and hepatitis B core-related antigen $(\mathrm{HBcrAg})$ has emerged as surrogate markers for evaluating covalently closed circular DNA [36], the unique template for pregenomic RNA transcription, and viral genome replication. Higher HBsAg and HBcrAg levels may suggest more active viral replication and hence a higher risk of HCC $[37,38]$. However, HBsAg and $\mathrm{HBcrAg}$ were not routinely quantified by the clinicians in this retrospective, multicenter study. Hence, we could not compare HBsAg and HBcrAg between HCC patients treated with ETV or TDF in the present study. However, in our recently published studies, we used HBsAg and HBcrAg to predict HBV relapse after ETV or TDF cessation $[39,40]$. Combining the data from both studies, 486 patients with CHB were treated with ETV ( $n$ $=301)$ or TDF $(n=185)$, with a median treatment duration of 157 months. We found that TDF had a higher potency in reducing HBcrAg than ETV (online suppl. Fig. 4). This result is not consistent with a recent study from Hong Kong [41], in which the magnitude of reduction of HBcrAg did not differ significantly between the ETV and TDF-treated groups after 2 years' treatment. However, the reduction in $\mathrm{HBcrAg}$ was numerically greater in the TDF-treated group. We believe that the long-term follow-up of serum HBcrAg could help to explain the differences between ETV and TDF observed in this study. Thus, further prospective, longitudinal studies that measure HBsAg and HBcrAg before HCC resection and annually are needed to confirm whether HBsAg and HBcrAg explain the differences in HCC recurrence between patients treated with ETV and TDF.

The anticancer effect of statins in HCC has been explored in vitro and in animal studies $[42,43]$. Many studies have recently revealed that statins exert protective effects in HCC and reduce HCC-related mortality [44, 45]. In this study, we also observed that statin use was associated with a lower incidence of tumor recurrence in patients with $\mathrm{HBV}$-related HCC after curative resection (HR, 0.236), although not significant in the multivariate
Tsai/Wang/Lee/Lin/Chang/Chen/Hung/ Lin/Hsiao/Chen/Chien/Hu 
analysis. This result is similar with our recently published study [46] and a meta-analysis [47], which showed that statin use was associated with reduced tumor recurrence in the HCC patients after curative resection. However, the proportions of patients taking statins were relatively small and all of the studies were retrospective; thus, numerous confounding factors could not be totally adjusted for. Thus, large, randomized trials of suitable regimens in well-selected patients treated using standard approaches are warranted to confirm the value of statins in HCC recurrence.

Another intriguing finding is that cigarette smoking is an independent risk factor for late recurrence in our HBV-related HCC cohort after resection with ETV or TDF treatment. Over 40 tobacco-related active compounds of cigarette smoke are metabolized and activated as carcinogens in the liver. Several studies reported the negative impacts of cigarette smoking and poor prognoses $[48,49]$. Furthermore, after surgery, continued smoking might be strongly correlated with tumor recurrence and compromised survival of HBV-related HCC patients [50]. Although there was no analysis about early or late recurrence in the study by Zhang et al. [50], we can see that the RFS among nonsmokers, ex-smokers, and current smokers started to deviate from 2 years after resection, which is compatible with our results.

Although our results are similar to those of a recently published article by Choi et al. [19], which enrolled the largest number of HCC patients to date, the present study has several strengths when compared to Choi's study. First, many factors including tumor burden, grade of differentiation, microvascular invasion, serum AFP, cirrhosis status, and HBV viral load are known to affect HCC recurrence. To minimize these confounding factors, we adopted strict inclusion criteria in the present study. For example, the modified BCLC stage, which excludes large single tumors $(>5 \mathrm{~cm})$ from stage A, was adopted to minimize the tumor burden effect. Furthermore, we recorded more potential risk factors, including alcohol consumption, smoking history, and the use of aspirin and statins in the analysis of the present study. Actually, these factors were not mentioned in the study by Choi et al. Second, we performed a comparison of on-therapy virological response at 1 or 2 years after surgery, which was not mentioned in the study by Choi et al. Third, as both studies were retrospective, heterogeneity must exist at the time of ETV or TDF initiation. To overcome this issue, we performed the subgroup analysis of NUC treatment-naïve patients after surgery. The results shows that the TDF group still had better RFS than the ETV group, which fur- ther supports our conclusion consistently. The analysis was not performed by Choi et al. Finally, we manually reviewed the medical records for each patient and checked their vital status using the Cancers Screening and Tracing Information Integrated System of Taiwan (https:// hosplab.hpa.gov.tw/CSTIIS/index.aspx). Thus, we could determine the exact status of every single patient enrolled in this study.

There are some potential limitations to this study. First, this was not a prospective study, and we could only collect data retrospectively from medical records. Despite employing PSM and multivariable analysis, not all confounding factors, such as HBV genotypes, gene mutations, $\mathrm{HBsAg}$, and $\mathrm{HBcrAg}$, which are associated with HCC development and recurrence [51-53], could be completely adjusted for analysis. Although unmeasured confounders may exist, we believe that the methodology used in the present study is solid and robust. Second, the number of patients in the TDF group was relatively low compared to the ETV group; however, the ratio of cases between ETV and TDF groups is comparable to the study from Taiwan [29] and reflects the real-world situation. Enrollment of larger number of patients in the TDF group is necessary in future studies. Finally, all of our patients were from Taiwan, where most patients with $\mathrm{CHB}$ are infected with genotype $\mathrm{B}$ or $\mathrm{C}$ hepatitis by vertical transmission. However, whether the effects discovered in this study could be generalized into Caucasian populations, where most patients are infected with genotype A or D by horizontal transmission, remain unclear. A validation cohort is necessary to confirm our major findings.

\section{Conclusion}

This study has demonstrated that TDF therapy is associated with a significantly lower risk of HCC recurrence, especially of late recurrence, than ETV therapy among patients who undergo curative hepatectomy for HBV-related early-stage HCC.

\section{Acknowledgments}

This work was supported by grants from Health and welfare surcharge of tobacco products, Ministry of Health and Welfare, Taiwan (MOHW107-TDU-B-212-114022, MOHW108-TDUB-212-124022, and MOHW109-TDU-B-212-134022 to C.-L. Chen). We acknowledge the Biostatistics Center, Kaohsiung Chang Gung Memorial Hospital for statistics work. 


\section{Statement of Ethics}

The clinical data were acquired with the approval and permission of the Institutional Review Board of the Kaohsiung Chang Gung Memorial Hospital. The study protocol was approved by the Institutional Review Board of the Kaohsiung Chang Gung Memorial Hospital. The written informed consent was waived according to the Institutional Review Board due to the retrospective design of the study with no relevant to human biological ethic problems.

\section{Conflict of Interest Statement}

The authors declare no conflicts of interest that pertain to this work.

\section{Funding Sources}

This research received no specific grant from any funding agency in the public, commercial, or not-for-profit sectors. The interpretation and reporting of the data were the sole responsibility of the authors.

\section{Author Contributions}

Ming-Chao Tsai and Tsung-Hui Hu were involved in conception and design; Ming-Chao Tsai contrbuted to the writing of the manuscript; Chih-Chi Wang, Wei-Chen Lee, Chih-Che Lin, KuoChin Chang, Ming-Tsung Lin, and Chao-Long Chen performed collection and assembly of data; and Chien-Hung Chen, ChaoHung Hung, Chang-Chun Hsiao, and Rong-Nan Chien performed data analysis or interpretation.

\section{Data Availability Statement}

All analyzed data are included in this published article. The original data are available upon reasonable request to the corresponding author.

\section{References}

1 Bray F, Ferlay J, Soerjomataram I, Siegel RL, Torre LA, Jemal A. Global cancer statistics 2018: GLOBOCAN estimates of incidence and mortality worldwide for 36 cancers in 185 countries. CA Cancer J Clin. 2018 Nov;68(6): 394-424.

2 Schweitzer A, Horn J, Mikolajczyk RT, Krause G, Ott JJ. Estimations of worldwide prevalence of chronic hepatitis B virus infection: a systematic review of data published between 1965 and 2013. Lancet. 2015 Oct 17; 386(10003):1546-55.

3 Altekruse SF, McGlynn KA, Reichman ME. Hepatocellular carcinoma incidence, mortality, and survival trends in the United States from 1975 to 2005. J Clin Oncol. 2009 Mar 20; 27(9):1485-91.

4 Wu JC, Huang YH, Chau GY, Su CW, Lai CR, Lee PC, et al. Risk factors for early and late recurrence in hepatitis B-related hepatocellular carcinoma. J Hepatol. 2009 Nov;51(5): $890-7$.

5 Tabrizian P, Jibara G, Shrager B, Schwartz M, Roayaie S. Recurrence of hepatocellular cancer after resection: patterns, treatments, and prognosis. Ann Surg. 2015 May;261(5):94755.

6 Hirokawa F, Hayashi M, Asakuma M, Shimizu T, Inoue Y, Uchiyama K. Risk factors and patterns of early recurrence after curative hepatectomy for hepatocellular carcinoma. Surg Oncol. 2016 Mar;25(1):24-9.

7 Kubo S, Hirohashi K, Tanaka H, Tsukamoto T, Shuto T, Yamamoto T, et al. Effect of viral status on recurrence after liver resection for patients with hepatitis B virus-related hepato- cellular carcinoma. Cancer. 2000 Mar 1;88(5): 1016-24.

8 Hung IF, Poon RT, Lai CL, Fung J, Fan ST, Yuen MF. Recurrence of hepatitis B-related hepatocellular carcinoma is associated with high viral load at the time of resection. Am J Gastroenterol. 2008 Jul;103(7):1663-73.

9 Wu CY, Chen YJ, Ho HJ, Hsu YC, Kuo KN, $\mathrm{Wu} \mathrm{MS}$, et al. Association between nucleoside analogues and risk of hepatitis B virus-related hepatocellular carcinoma recurrence following liver resection. JAMA. 2012 Nov 14; 308(18):1906-14.

10 Yin J, Li N, Han Y, Xue J, Deng Y, Shi J, et al. Effect of antiviral treatment with nucleotide/ nucleoside analogs on postoperative prognosis of hepatitis B virus-related hepatocellular carcinoma: a two-stage longitudinal Clinical Study. J Clin Oncol. 2013 Oct 10;31(29):3647-55.

11 Sarin SK, Kumar M, Lau GK, Abbas Z, Chan HL, Chen CJ, et al. Asian-Pacific clinical practice guidelines on the management of hepatitis B: a 2015 update. Hepatol Int. 2016 Jan; 10(1):1-98

12 European Association for the Study of the Liver. EASL 2017 Clinical Practice Guidelines on the management of hepatitis B virus infection. J Hepatol. 2017 Aug;67(2):370-98.

13 Terrault NA, Lok ASF, McMahon BJ, Chang KM, Hwang JP, Jonas MM, et al. Update on prevention, diagnosis, and treatment of chronic hepatitis B: AASLD 2018 hepatitis B guidance. Hepatology. 2018 Apr;67(4):156099.

14 Qi X, Li J, Deng H, Li H, Su C, Guo X. Neutrophil-to-lymphocyte ratio for the prognos- tic assessment of hepatocellular carcinoma: a systematic review and meta-analysis of $\mathrm{Ob}$ servational Studies. Oncotarget. 2016 Jul 19; 7(29):45283-301.

15 Papatheodoridis GV, Idilman R, Dalekos GN, Buti M, Chi H, van Boemmel F, et al. The risk of hepatocellular carcinoma decreases after the first 5 years of entecavir or tenofovir in caucasians with chronic hepatitis B. Hepatology. 2017 Nov;66(5):1444-53.

16 Choi J, Kim HJ, Lee J, Cho S, Ko MJ, Lim YS Risk of hepatocellular carcinoma in patients treated with entecavir vs tenofovir for chronic hepatitis B: a Korean Nationwide Cohort Study. JAMA Oncol. 2019 Jan 1;5(1): 30-6.

17 Zhang Z, Zhou Y, Yang J, Hu K, Huang Y. The effectiveness of TDF versus ETV on incidence of HCC in CHB patients: a meta analysis. BMC Cancer. 2019 May 29;19(1):511.

$18 \mathrm{Hu}$ TH, Yueh-Hsia Chiu S, Tseng PL, Chen $\mathrm{CH}, \mathrm{Lu} \mathrm{SN}$, Wang JH, et al. Five-year comparative risk of hepatocellular carcinoma development under entecavir or tenofovir treatment-naïve patients with chronic hepatitis Brelated compensated cirrhosis in Taiwan. Aliment Pharmacol Ther. 2020 Dec;52(1112):1695-706.

19 Choi J, Jo C, Lim YS. Tenofovir versus entecavir on recurrence of hepatitis $B$ virus-related hepatocellular carcinoma after surgical resection. Hepatology. 2021 Feb;73(2):661-73.

20 European Association for the Study of the Liver. EASL clinical practice guidelines: management of hepatocellular carcinoma. J Hepatol. 2018 Jul;69(1):182-236. 
21 Heimbach JK, Kulik LM, Finn RS, Sirlin CB, Abecassis MM, Roberts LR, et al. AASLD guidelines for the treatment of hepatocellular carcinoma. Hepatology. 2018 Jan;67(1):35880.

22 European Association for the Study of the Liver, European Organisation for Research and Treatment of Cancer. EASL-EORTC clinical practice guidelines: management of hepatocellular carcinoma. Eur J Cancer. 2012 Apr;48(4):599-641.

23 Bruix J, Sherman M; American Association for the Study of Liver Disease. Management of hepatocellular carcinoma: an update. Hepatology. 2011 Mar;53(3):1020-2.

24 Edmondson HA, Steiner PE. Primary carcinoma of the liver: a study of 100 cases among 48,900 necropsies. Cancer. 1954 May;7(3): 462-503.

25 Ishak K, Baptista A, Bianchi L, Callea F, De Groote J, Gudat F, et al. Histological grading and staging of chronic hepatitis. J Hepatol. 1995 Jun;22(6):696-9.

26 World Health Organization. WHO collaborating centre for drug statistics methodology, guidelines for ATC classification and DDD assignment 2020. Oslo, Norway: World Health Organization; 2020.

27 Heagerty PJ, Lumley T, Pepe MS. Time-dependent ROC curves for censored survival data and a diagnostic marker. Biometrics. 2000 Jun;56(2):337-44.

28 Imamura $\mathrm{H}$, Matsuyama $\mathrm{Y}$, Tanaka E, Ohkubo T, Hasegawa K, Miyagawa S, et al. Risk factors contributing to early and late phase intrahepatic recurrence of hepatocellular carcinoma after hepatectomy. J Hepatol. 2003 Feb; 38(2):200-7.

29 Wu CJ, Chau GY, Lee IC, Huo TI, Su CW, Hou MC, et al. Early and late recurrence of surgically resected hepatitis B virus-related hepatocellular carcinoma on nucleos(t)ide analogues therapy. J Formos Med Assoc. 2021 Aug;120(8):1563-71.

30 Lok AS, McMahon BJ, Brown RS Jr, Wong JB, Ahmed AT, Farah W, et al. Antiviral therapy for chronic hepatitis B viral infection in adults: a systematic review and meta-analysis. Hepatology. 2016 Jan;63(1):284-306.

31 Tseng CH, Tseng CM, Wu JL, Hsu YC, El-Serag HB. Magnitude of and prediction for risk of hepatocellular carcinoma in patients with chronic hepatitis B taking entecavir or tenofovir therapy: a systematic review. J Gastroenterol Hepatol. 2020 Oct;35(10):1684-93.

32 Ke W, Liu L, Zhang C, Ye X, Gao Y, Zhou S, et al. Comparison of efficacy and safety of te- nofovir and entecavir in chronic hepatitis B virus infection: a systematic review and metaanalysis. PLoS One. 2014;9(6):e98865.

33 Gao L, Trinh HN, Li J, Nguyen MH. Tenofovir is superior to entecavir for achieving complete viral suppression in $\mathrm{HBeAg-positive}$ chronic hepatitis B patients with high HBV DNA. Aliment Pharmacol Ther. 2014 Mar; 39(6):629-37.

34 Murata K, Asano M, Matsumoto A, Sugiyama M, Nishida N, Tanaka E, et al. Induction of IFN-lambda3 as an additional effect of nucleotide, not nucleoside, analogues: a new potential target for HBV infection. Gut. 2018 Feb; 67(2):362-71.

35 Brambilla G, Mattioli F, Robbiano L, Martelli A. Studies on genotoxicity and carcinogenicity of antibacterial, antiviral, antimalarial and antifungal drugs. Mutagenesis. 2012 Jul; 27(4):387-413.

36 Testoni B, Lebossé F, Scholtes C, Berby F, Miaglia C, Subic M, et al. Serum hepatitis B core-related antigen ( $\mathrm{HBcrAg}$ ) correlates with covalently closed circular DNA transcriptional activity in chronic hepatitis B patients. J Hepatol. 2019 Apr;70(4):615-25.

37 Tseng TC, Liu CJ, Yang HC, Su TH, Wang CC, Chen CL, et al. High levels of hepatitis B surface antigen increase risk of hepatocellular carcinoma in patients with low HBV load. Gastroenterology. 2012 May;142(5):1140-4.

38 Tseng TC, Liu CJ, Hsu CY, Hong CM, Su TH, Yang WT, et al. High level of hepatitis B corerelated antigen associated with increased risk of hepatocellular carcinoma in patients with chronic HBV infection of intermediate viral load. Gastroenterology. 2019 Dec;157(6): 1518-29.e3.

39 Huang PY, Wang JH, Hung CH, Lu SN, Hu $\mathrm{TH}$, Chen $\mathrm{CH}$. The role of hepatitis $\mathrm{B}$ virus core-related antigen in predicting hepatitis $\mathrm{B}$ virus relapse after cessation of entecavir in hepatitis B e antigen-negative patients. J Viral Hepat. 2021 Aug;28(8):1141-9.

40 Kuo YH, Wang JH, Hung CH, Lu SN, Hu TH, $\mathrm{Chen} \mathrm{CH}$. Combining end-of-treatment $\mathrm{HB}$ sAg and baseline hepatitis B core-related antigen reduce $\mathrm{HBV}$ relapse rate after tenofovir cessation. Hepatol Int. 2021 Apr;15(2):301-9.

41 Mak LY, Wong DK, Cheung KS, Seto WK, Fung J, Yuen MF. First-line oral antiviral therapies showed similar efficacies in suppression of serum $\mathrm{HBcrAg}$ in chronic hepatitis B patients. BMC Gastroenterol. 2021 Mar 17;21(1):123.

42 Urbich C, Dernbach E, Zeiher AM, Dimmeler $S$. Double-edged role of statins in angiogene- sis signaling. Circ Res. 2002 Apr 5;90(6):73744.

43 Demierre MF, Higgins PD, Gruber SB, Hawk E, Lippman SM. Statins and cancer prevention. Nat Rev Cancer. 2005 Dec;5(12):930-42.

44 Tsan YT, Lee CH, Wang JD, Chen PC. Statins and the risk of hepatocellular carcinoma in patients with hepatitis B virus infection. J Clin Oncol. 2012 Feb 20;30(6):623-30.

45 Tsan YT, Lee CH, Ho WC, Lin MH, Wang JD, Chen PC. Statins and the risk of hepatocellular carcinoma in patients with hepatitis $\mathrm{C}$ virus infection. J Clin Oncol. 2013 Apr 20; 31(12):1514-21.

46 Yang SY, Wang CC, Chen KD, Liu YW, Lin $\mathrm{CC}$, Chuang $\mathrm{CH}$, et al. Statin use is associated with a lower risk of recurrence after curative resection in BCLC stage 0-A hepatocellular carcinoma. BMC Cancer. 2021 Jan 15;21(1): 70.

47 Li X, Liu L, Hu Y. Statin use and the prognosis of patients with hepatocellular carcinoma: a meta-analysis. Biosci Rep. 2020 Apr 30;40(4): 40.

48 Chuang SC, Lee YC, Hashibe M, Dai M, Zheng T, Boffetta P. Interaction between cigarette smoking and hepatitis $\mathrm{B}$ and $\mathrm{C}$ virus infection on the risk of liver cancer: a metaanalysis. Cancer Epidemiol Biomarkers Prev. 2010 May;19(5):1261-8.

49 Shih WL, Chang HC, Liaw YF, Lin SM, Lee $\mathrm{SD}$, Chen PJ, et al. Influences of tobacco and alcohol use on hepatocellular carcinoma survival. Int J Cancer. 2012 Dec 1;131(11):261221.

50 Zhang XF, Wei T, Liu XM, Liu C, Lv Y. Impact of cigarette smoking on outcome of hepatocellular carcinoma after surgery in patients with hepatitis B. PLoS One. 2014;9(1): e85077.

51 Huang G, Lau WY, Zhou WP, Shen F, Pan ZY, Yuan SX, et al. Prediction of hepatocellular carcinoma recurrence in patients with low hepatitis B virus DNA levels and high preoperative hepatitis B surface antigen levels. JAMA Surg. 2014 Jun;149(6):519-27.

52 Chen S, Jia J, Gao Y, Li H, Fang M, Feng H, et al. Clinical evaluation of hepatitis B core-related antigen in chronic hepatitis B and hepatocellular carcinoma patients. Clin Chim Acta. 2018 Nov;486:237-44.

53 Yen CJ, Ai YL, Tsai HW, Chan SH, Yen CS, Cheng $\mathrm{KH}$, et al. Hepatitis B virus surface gene pre-S2 mutant as a high-risk serum marker for hepatoma recurrence after curative hepatic resection. Hepatology. 2018 Sep; 68(3):815-26. 